Universidade Tecnológica Federal do Paraná - UTFPR Campus Ponta Grossa - Paraná - Brasil ISSN: 1981-3686/v. 04, n. 02: p. 207-217, 2010 DOI: $10.3895 / \mathrm{S} 1981-36862010000200009$
Revista Brasileira de Tecnologia

Agroindustrial

\title{
DESTINAÇÃO FINAL DOS RESÍDUOS SÓLIDOS DE SERVIÇOS DE SAÚDE EM PEQUENAS PROPRIEDADES RURAIS DA COLÔNIA WITMARSUM-PR
}

\section{DESTINATION WAYS OF HEALTH SERVICE SOLID WASTE (HSSW) IN SMALL FARMS ON WITMARSUM COLONY-PR}

\author{
Adeline Chaicouski ${ }^{1}$; Joyce Estina da Silva ${ }^{2}$; Suélyn Bomfim Nigelski ${ }^{3}$; \\ Rosane Yoshida Natume ${ }^{4}$; Isonel Sandino Meneguzzo ${ }^{5}$ \\ ${ }^{1}$ Universidade Tecnológica Federal do Paraná - UTFPR - Ponta Grossa chaicouski@ hotmail.com \\ ${ }^{2}$ Universidade Tecnológica Federal do Paraná - UTFPR - Ponta Grossa joyceestina@ hotmail.com \\ ${ }^{3}$ Universidade Tecnológica Federal do Paraná - UTFPR - Ponta Grossa sunigelski@ hotmail.com \\ ${ }^{4}$ Universidade Tecnológica Federal do Paraná - UTFPR - Ponta Grossa rnatume_utfpr@yahoo.com.br \\ ${ }^{5}$ Centro de Ensino Superior dos Campos Gerais - CESCAGE - Ponta Grossa imeneguzzo@hotmail.com
}

\begin{abstract}
Resumo
Este texto tem como objetivo caracterizar a forma de destinação final dos resíduos sólidos de serviços de saúde (RSSS), de origem veterinária, na Colônia Witmarsum, localizada na zona rural do município de Palmeira, PR. O risco potencial ligado ao manejo dos RSSS aumenta a necessidade de implantação de programas de gerenciamento mais eficazes, para garantir a segurança da população, do meio ambiente, além da saúde dos próprios trabalhadores. O presente trabalho foi desenvolvido durante os meses de setembro a novembro de 2009 e a metodologia adotada para verificar a situação dos produtores foram visitas e entrevistas. Os resultados permitiram afirmar que a falta do PGRSSS é um dos vários problemas enfrentados por pequenos produtores, pois como não há programa de coleta de lixo, os resíduos de medicamentos veterinários são dispostos a céu aberto, armazenados em sacos plásticos, retidos na própria propriedade e em alguns casos, levados até o "lixão" da Colônia.
\end{abstract}

Palavras-chave: resíduos sólidos de serviços de saúde; gerenciamento de resíduos; Colônia Witmarsum.

\section{Introdução}

O tema qualidade ambiental, não só no Brasil, tornou-se debatido em todos os meios, devido à crescente degradação ambiental. O lixo vem se tornando um dos maiores problemas enfrentados no mundo e a questão dos resíduos sólidos surge como uma séria ameaça à população. Scarlato (1992) 
explica que, por mais contraditório que pareça, o homem dito inteligente, vem colocando em seu habitat resíduos da civilização, sendo assim a espécie humana fortemente competidora. Os resíduos sólidos resultam de atividades humanas diversas (industriais, agropecuária, construção civil, público, tóxicos, radioativos, domiciliares, tecnológicos e de serviços de saúde) e que, dependendo da sua composição, podem contaminar o meio ambiente (BRASIL, 2004).

Quando se trata sobre resíduos, em geral refere-se às embalagens sem utilidade após o consumo de seus produtos ou aos produtos que não atendem mais aos objetivos pelos quais foram criados. De acordo com a NBR 10.004 (2004), definem-se como resíduos sólidos, ou simplesmente lixo, todo e qualquer material sólido proveniente das atividades diárias do homem em sociedade, cujo produtor ou proprietário não considere com valor suficiente para sua conservação.

A questão dos resíduos de serviços de saúde não deve apenas ser analisada no aspecto da transmissão de doenças, mas também está envolvida a questão da saúde do trabalhador e a conservação do meio ambiente, o que envolve questões de biossegurança (GARCIA e ZANETTI-RAMOS, 2004).

Os RSSS podem ser provenientes de grandes geradores tais como indústrias químicofarmacêuticas, hospitais, ou pequenos geradores como residências, inclusive os provenientes de atividades agropecuárias. Os pequenos produtores normalmente não são muito fiscalizados, por serem considerados não-impactantes. O risco potencial ligado ao manejo dos RSSS aumenta a necessidade de implantação de programas de gerenciamento mais eficazes, para garantir a segurança e saúde dos trabalhadores, população e do meio ambiente (RODRIGUES, 2006).

De acordo com Tenório e Espinosa (2004) os Resíduos Sólidos de Serviço de Saúde (RSSS) são aqueles gerados em hospitais, clínicas veterinárias, laboratórios, farmácias, entre outros. Esses resíduos podem ser classificados em Comuns, representados por papéis, invólucros, como exemplo e os Sépticos, que exigem maior cuidado devido ao potencial risco de contaminação (TENÓRIO e ESPINOSA, 2004).

Segundo a Resolução n 283 , de 12 de julho de 2001 - CONAMA - os Resíduos de Serviços de Saúde são [1] derivados de qualquer unidade que efetue atividades de caráter médico-assistencial de origem humana ou animal; [2] derivados de centros de pesquisa, desenvolvimento ou experimentação nas áreas de farmacologia e saúde; [3] medicamentos e imunoterápicos deteriorados ou vencidos; [4] provenientes de necrotérios, funerárias ou quaisquer serviços de medicina legal; [5] derivados de barreiras sanitárias. 
A Agência de Vigilância Sanitária - ANVISA - classifica os RSSS de acordo com a sua natureza, pela RDC n 306 de 7 de dezembro de 2004, em cinco categorias, como apresentados no quadro 1.

Quadro 1: Distribuição dos Resíduos Sólidos de acordo com o tipo

\begin{tabular}{|c|c|}
\hline Categoria & Tipo de Resíduo \\
\hline Tipo A & Resíduos com risco biológico \\
\hline Tipo B & Resíduos com risco químico \\
\hline Tipo C & Resíduos radioativos \\
\hline Tipo D & Comuns e não recicláveis \\
\hline Tipo E & Resíduos perfurocortantes \\
\hline
\end{tabular}

Fonte: ANVISA, 2004

Os resíduos do grupo A são aqueles que apresentam agentes biológicos. O grupo B é caracterizado por conter substâncias químicas inflamáveis, corrosivos, reativo e tóxico. No grupo C, encontram-se os resíduos provenientes de atividade humana com radionuclídeos em quantidades superiores a limites estabelecidos por lei. A maioria dos resíduos que não apresentam risco à saúde e ao meio ambiente enquadra-se no grupo D e os materiais perfurocortantes (bisturi, ampolas, vidros, entre outros), no grupo E (SPINA, 2005). A maioria dos resíduos de medicamentos veterinários se enquadra no grupo E, no qual estão incluídos os materiais perfurocortantes.

Os RSSS podem conter microrganismos patogênicos, entre os quais bactérias, fungos e vírus, sendo potenciais transmissores de doenças (TENÓRIO e ESPINOSA, 2004), devendo ser tratados adequadamente para reduzir ou eliminar a sua carga microbiana e se tornarem menos perigosos. Entretanto, alguns autores não consideram os RSSS como perigosos. De acordo com Zanon (1990) não há evidências científicas que comprovem a relação entre aquisição de doença pelo contato com o resíduo, e esclarece que são necessários fatores para adoecer tais como o patógeno, a dose de inoculação e a suscetibilidade do hospedeiro.

Para realizar a coleta destes resíduos seguem-se os padrões estabelecidos na legislação (SILVA e SOARES, 2004), como a Resolução CONAMA n ${ }^{\circ}$ 306, de 7 de dezembro de 2004, que estabelece o Regulamento Técnico para o Gerenciamento de Resíduos de Serviços de Saúde para todos os estabelecimentos geradores de RSSS, e a Resolução CONAMA n 283, de 12 de julho de 2001, que determina as normas para o tratamento e destinação final dos RSSS. 
Os materiais perfurocortantes devem ser descartados separadamente, no local de geração, logo após o uso, em recipientes, rígidos, resistentes a ruptura e vazamento, com tampa, devidamente identificados, sendo expressamente proibido o esvaziamento desses recipientes para reaproveitamento. As agulhas descartáveis devem ser desprezadas juntamente com as seringas. Os resíduos perfurocortantes contaminados com agentes biológicos devem ser tratados, utilizando-se processo físico ou outros processos que reduzam eliminem a carga microbiana. Seringas e agulhas utilizadas em de amostras e demais resíduos perfurocortantes não necessitam de tratamento (MPGRSS, 2004).

Antes de sua destinação final, os RSSS devem ser processados em sua origem, separando-se o material contaminado daquele que não está, para que destinação correta posterior. Esses materiais devem ser colocados em sacos de cor leitosa marcados com o símbolo de material infectante (figura 1) e a inscrição "resíduo perfurocortante" (NBR 7.500:2003). A disposição ou destino final de RSSS é o confinamento destes em valas sépticas ou em aterro sanitário, após a incineração (SPINA, 2005). Nas valas os RSSS são tratados por reação exotérmica (água + cal virgem) e posteriormente cobertos com terra (BRASIL, 2001). Essas valas sépticas logo se tornam passivos ambientais, deixando a área onde se encontram improdutivas por anos ou décadas, e ainda, devido a sua carga contaminante, se tornam agentes poluidores de ar, água e solo (SPINA, 2005). O tratamento mais freqüente é a incineração, com as cinzas destinadas a aterros sanitários (TENÓRIO e ESPINOSA, 2004), embora existam outros métodos como autoclave, desinfecção química, radiação ou ionização (SPINA, 2005).

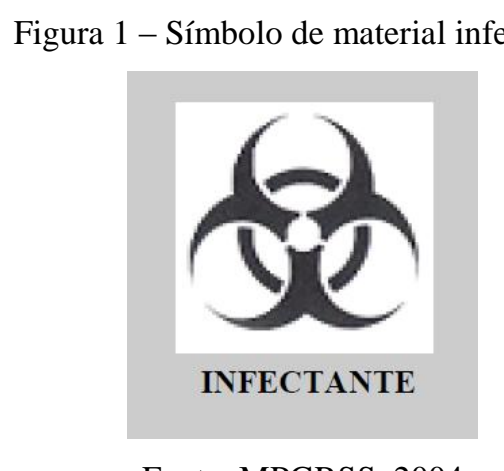

Fonte: MPGRSS, 2004

A crescente utilização dos serviços de saúde levou a geração de resíduos sólidos de saúde (RSSS) e com a Resolução 306/2004 da ANVISA, o setor passou a ser responsável pela destinação final desses resíduos (RODRIGUES, 2006). 
Tendo em vista a falta de um Programa de Gerenciamento dos Resíduos de Serviço de Saúde (RSSS) na Colônia Witmarsum, localizada na área rural do município de Palmeira- PR, este trabalho objetivou verificar a adequação quanto à legislação vigente com relação ao gerenciamento dos medicamentos veterinários utilizados pelos pecuaristas dessa comunidade, visando fornecer dados para implantação futura de um sistema adequado de destinação final.

\section{Material e Métodos}

A Colônia Witmarsum foi constituída em julho de 1951 por menonitas vindos da cidade de Witmarsum do estado de Santa Catarina. Os colonos de Witmarsum pertencem ao grupo dos menonitas alemães-russos, que tem sua origem na Frísia, no norte da atual Holanda e Alemanha. Através da Prússia eles migraram para Rússia no século XVIII, de onde fugiram em 1929, quando o comunismo se instalou naquele país. Em 1930 vieram para o Brasil onde, após um tempo de permanência em Santa Catarina, uma parte destes mudou-se para o estado do Paraná (ACMPW, 2009).

A colônia possui uma área de aproximadamente 7800 hectares e aproximadamente 3000 habitantes. Compreende cinco núcleos de povoamento, denominados aldeias/glebas numeradas de 1 a 5 e, dispostas em torno de um centro administrativo comercial e social. Atualmente, as propriedades são individuais, com lotes rurais de 50 ha em média. Localizada a cerca de $23 \mathrm{Km}$ do municipío de Palmeira, 62 Km de Ponta Grossa e 60 Km de Curitiba (figura 2), está ligada à BR 277 e à BR 376 por uma estrada estadual asfaltada. Sua base econômica reside na agropecuária, desenvolvida sobretudo no setor da pecuária leiteira, apresentando um total de setenta e oito pecuaristas. Também há criação de frangos e suínos, sendo respectivamente onze e três pecuaristas, além do cultivo de soja e milho. Nos

últimos anos apresenta um crescimento considerável relacionado às atividades turísticas (ACMPW, 2009).

Este trabalho foi desenvolvido entre os meses de setembro a novembro de 2009. O trabalho de campo foi realizado na Colônia Witmarsum, nos dias 16, 17 e 18 de outubro de 2009, para coleta de dados. Visitas foram feitas a dois produtores de leite, um criador de suínos, um de frangos, e um criador de cães. Todos esses ao trabalharem com animais fazem uso de medicamentos veterinários, sem destinação final adequada após o uso dos mesmos. A coleta das informações foi feita por meio de conversa informal, anotando-se os pontos de maior importância para cada criador. 


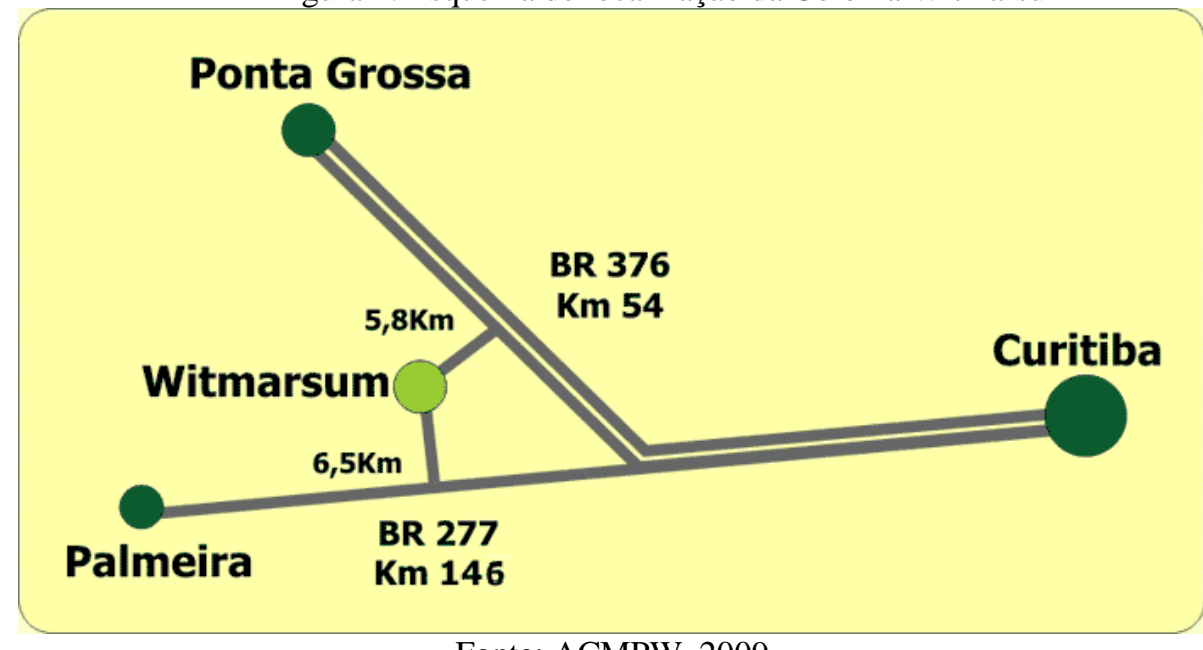

Fonte: ACMPW, 2009

Sem escala

\section{Resultados e Discussão}

Após a realização da visita, entrevista e análise dos dados coletados, verificou-se que a falta de um programa de coleta dos RSSS é mais um dos problemas ambientais enfrentados pelos pecuaristas de Witmarsum. A colônia possui uma área relativamente pequena e depende de diversos serviços do poder público do município de Palmeira.

Destaca-se que o governo municipal parece não demonstrar interesse na coleta e no gerenciamento dos resíduos gerados nesta localidade ${ }^{1}$. Embora a legislação ambiental seja rigorosa, nenhum dos pecuaristas visitados relatou ter problemas com a fiscalização do Ministério da Agricultura e Pecuária. O tratamento e a destinação final dos resíduos sólidos em geral, ainda se resumem na adoção de soluções imediatistas, quase sempre fundamentadas no simples descarte, predominando os depósitos a céu aberto que contribuem para a degradação ambiental. Dos cinco pecuaristas visitados, todos desconheciam o que deve ser feito com os resíduos de medicamentos veterinários usados nos animais, indicando que não houve orientação adequada referente aos RSSS, na colônia. A tabela 1 mostra o número de pecuaristas visitados e suas respectivas produções, assim como as soluções encontradas para o descarte dos RSSS pelos pecuaristas visitados na Colônia Witmarsum, que foram bem diversificadas. 
Tabela 1- Produção pecuária e as soluções encontradas para destinação final dos RSSS

\begin{tabular}{cccc}
\hline Proprietário & Animais & Produção & Solução \\
\hline 01 & Bovinos & Leite & Descarta junto com dejetos orgânicos humanos ${ }^{2}$ \\
02 & Bovinos & Leite & Armazena em sacos plásticos no sótão da casa \\
03 & Frango & Animais para abate & Retidos na propriedade \\
04 & Suínos & Animais para abate & Retidos na propriedade \\
05 & Cães & Venda de animais & Leva até o "lixão" da colônia \\
\hline
\end{tabular}

FONTE: Pesquisa de campo

Dois dos pecuaristas visitados adquirem os medicamentos no mesmo ponto de venda, na cooperativa Witmarsum, e o criador de cães compra em Curitiba, pois os medicamentos veterinários vendidos na colônia são exclusivamente para bovinos, suínos e frangos. Os criadores de frango e suíno recebem os medicamentos da Perdigão e já solicitaram que a empresa faça o recolhimento das embalagens de medicamentos veterinários, uma vez que estes animais são vendidos para esta empresa. Todos afirmaram fazer uso dos medicamentos somente quando necessário, para tratar doenças comuns dos animais, que não ocorrem com freqüência.

"Melhor do que tratar é prevenir", citou um dos produtores de leite durante a realização da visita, que faz uso de medicamentos homeopáticos na alimentação dos animais, o que reduz o uso de medicamentos veterinários. Este relatou ainda que somente as embalagens de agrotóxicos são recolhidas na propriedade, assim como nas demais propriedades. Nenhum dos pecuaristas sabia qual o procedimento para descarte das ampolas de injeções e vidros de medicamentos.

Conforme consta na Tabela 1, as soluções adotadas pelos proprietários geram um risco potencial para o ambiente e para os seres humanos, pois pode haver a contaminação do lençol freático e do solo e crianças e/ou adolescentes entrarem em contato com algum tipo de resíduo disposto a céu aberto.

Um aspecto que merece destaque é o que os moradores locais denominam de "lixão" da Colônia. Este constitui-se numa área com relevo plano, com vegetação rasteira e afastada das residências locais. Cabe salientar que, em todas as propriedades visitadas, houve interesse por parte dos pecuaristas em desenvolver um Programa de Gerenciamento, não só para os resíduos de serviço de saúde, mas também para outros tipos de resíduos. Destaca-se ainda que a produção orgânica origina

\footnotetext{
${ }^{1}$ A colônia Witmarsum não possui coleta de lixo. Os moradores e produtores se encarregam de dar uma destinação para os resíduos gerados, desde a incineração, descarte a céu aberto e abertura de valas para enterrarem seus próprios resíduos.

${ }_{2}$ Privada ou casinha: Espécie de banheiro externo, muito comum em propriedades rurais. Apresenta uma fossa cavada na terra onde os dejetos ficam depositados.
} 
menos resíduos veterinários, sendo assim uma ótima alternativa de minimização do problema, causando menos impactos no ambiente, além de garantir alimentos saudáveis livres de contaminantes.

A contaminação do meio ambiente por resíduos sejam eles sólidos, líquidos ou gasosos, causa efeitos no ser humano, tanto direta quanto indiretamente, via ar, água ou alimentos. Uma vez contaminados, os malefícios são transferidos para toda a cadeia biológica (BRASIL, 2004). A importância de um gerenciamento correto dos RSSS é nítida e são várias as publicações na área. Desta forma se faz necessária a implantação de um Programa de Gerenciamento de Resíduos Sólidos de Serviço de Saúde (PGRSSS) em todas as unidades geradoras.

A Colônia Witmarsum necessita de um programa de gerenciamento para os resíduos de saúde, visto que os medicamentos de uso veterinário utilizados nos animais não apresentam destinação final adequada, devido, não só a falta de informação, mas sim de uma participação do Poder Público, ao qual a colônia está vinculada.

Os resultados da pesquisa permitiram afirmar que a falta do PGRSSS é um dos vários problemas enfrentados por pequenos produtores, pois como não há nenhum programa de coleta de lixo, os resíduos de medicamentos veterinários são descartados de forma incorreta. O Programa de Gerenciamento dos Resíduos de Saúde deve ter envolvimento coletivo, ou seja, deve ser realizado em conjunto com todos os interessados (RODRIGUES, 2006). O pessoal envolvido no gerenciamento dos resíduos deve ser capacitado na ocasião de sua admissão e mantido sob treinamento periódico, pois todos que manuseiam os RSSS estão expostos a riscos quando esses resíduos são mal gerenciados (GARCIA e ZANETTI-RAMOS, 2004). Tendo em vista a necessidade de um PGRSSS, a boa vontade dos produtores, em participar ativamente do programa e a iniciativa de alunos de graduação, com a base técnico-científica, falta apenas a participação do Poder Público, no sentido de colaborar para a melhoria contínua da qualidade de vida dos moradores da Colônia Witmarsum. Os resíduos dos serviços de saúde merecem uma atenção especial desde a sua geração até a sua disposição final, pois constituem potencial risco para os seres humanos e para o ambiente.

\section{Conclusões}

O gerenciamento dos RSSS na área pesquisada não ocorre de forma adequada tal como preconiza a Resolução RDC n 306/04 e a Resolução CONAMA n 283/01.

Medidas corretas devem ser tomadas em caráter de urgência na Colônia, por parte da sociedade civil organizada e do poder público municipal no sentido de levar conhecimentos técnico-científicos 
aos pecuaristas para que os mesmos possam dar uma destinação adequada aos resíduos de origem veterinária.

\begin{abstract}
This article has to purpose to characterize the destination ways of Health Service Solid Waste (HSSW) in Witmarsum Colony, located in the rural area of the local authority of Palmeira, PR. The potential risk related HSSW Management show the need to implement management programs more effective, to ensure the safety to population, environment beyond own workers' health. This study was conducted during September to November 2009 and the methodology adopted to check the circumstances producers', were visits, interviews and analysis of the collected data. It was verified that the lack of a HSSW collection program is a problem for farmers. The results allowed to affirm that the lack of the is one of the several problems faced by small producers, since as there isn't program of collection of garbage, the veterinary medicines residues are discarded in the incorrect form, different alternatives are adopting like exposure to open air, burn and bury.
\end{abstract}

Key-words: health services solid waste, waste management, Witmarsum Colony.

\title{
Referências
}

ABNT - ASSOCIAÇÃO BRASILEIRA DE NORMAS TÉCNICAS. NBR 7.500:2003 - Identificação para o transporte terrestre, manuseio, movimentação e armazenamento de produtos, disponível em http://www.scribd.com/doc/35717137/NBR-7500>. Acesso em 26 nov. 2010.

$\begin{array}{cccccc}\text { · } & \text { NBR } & 10.004: 2004 & \text { Resíduos } & \text { sólidos-classificação, } & \text { disponível }\end{array}$

ACMPW - ASSOCIAÇÃO COMUNITÁRIA DOS MORADORES PROPRIETÁRIOS DE WITMARSUM. Localização, disponível em <http://www.acmpw.com.br/turismo/localiza.htm> Acesso em 17 out. 2009.

Seja bem vindo a Colônia Witmarsum. Disponível em <http://www.acmpw.com.br/turismo/index.php> acesso em 17 out. 2009.

AGÊNCIA NACIONAL DE VIGILÂNCIA SANITÁRIA - ANVISA. Resolução RDC n 206, de 7 de dezembro de 2004. Regulamento técnico para o gerenciamento de resíduos de serviço de saúde, disponível em <http://elegis.anvisa.gov.br/leisref/public/showAct.php?id=13554>. Acesso em 16 set. 2009.

ANDRADE, J. B. L. de. Determinação da composição gravimétrica dos Resíduos de Serviços de Saúde de diferentes tipos de estabelecimentos geradores. $20^{\circ}$ Congresso Brasileiro de Engenharia Sanitária e Ambiental, disponível em: <http://www.bvsde.paho.org//bvsaidis//brasil20//iii-007.pdf>. Acesso em 18 set.2009

BRASIL, A. M.; SANTOS, F. SIMÃO, L. K. Equilíbrio ambiental e Resíduos na sociedade moderna. São Paulo: FAARTE, 2004.

BRASIL. Ministério da Saúde. Gerenciamento de Resíduos de serviço de Saúde. Projeto Reforsus, Brasília, 2001.

Resolução da Diretoria Colegiada $\mathbf{n}^{\mathbf{0}}$ 306, de 7 de dezembro de 2004, disponível em <http://www.unifesp.br/reitoria/residuos/legislacao/arquivos/RDC_306_ANVISA.pdf>. Acesso em 16 set.2009.

CONAMA - CONSELHO NACIONAL DO MEIO AMBIENTE -. Resolução no 283, de 12 de julho de 2001. Tratamento e a destinação final dos resíduos dos serviços de saúde, disponível em <http://elegis.anvisa.gov.br/leisref/public/showAct.php?id=17972eword=>. Acesso em 16 set. 2009. 
GARCIA, L. P; ZANETTI-RAMOS, B. G. Gerenciamento dos resíduos de serviços de saúde: uma questão de biossegurança. Revista Caderno de Saúde Pública, v. 20, n. 3, p. 744-752, 2004.

LISBOA JR, A. F. Responsabilidade Sócio Ambiental em Saúde - Legislação Sanitária e Ambiental - 2009. Gerenciamento dos Resíduos de Serviços de Saúde, disponível em <http://www.crasp.com.br/convivencia/artigos_saude/evento_17_06_09/apresentacao.ppt>. Acesso em 18 set. 2009.

MGRSS - Manual de gerenciamento de resíduos de serviços de saúde, disponível em <http://www.visa.goias.gov.br/arquivos/manual_PGRSS.pdf>. Acesso 26 nov. 2010.

RODRIGUES, C. R. B. Abordagem dos resíduos sólidos de serviços de saúde na formação acadêmica em cursos da área da saúde. 58 f. Monografia (Especialização em Gestão Industrial). UTFR- Campus Ponta Grossa, Ponta Grossa, 2006.

SCARLATO, F.C. Do nicho ao lixo: ambiente, sociedade e educação. (Série Meio Ambiente) São Paulo: Atual, 1992.

SILVA, R. F. S; SOARES, M. L. Gestão dos resíduos sólidos de serviços de saúde com responsabilidade social. VII SEMEAD 2004 - RELATO DE EXPERIÊNCIA GESTÃO SOCIOAMBIENTAL, disponível em <http://www.ead.fea.usp.br/semead/7semead/paginas/artigos\%20recebidos/Socioambiental/SA25_Gest\%E3o_dos_res\%ED duos_solidos.PDF>. Acesso em 18 set. 2009.

SPINA, M. I. A. P. Características do gerenciamento dos resíduos sólidos dos serviços de saúde em Curitiba e análise das implicações socioambientais decorrentes dos métodos de tratamento e destino final. Revista RA'EGA, Curitiba, n. 9, p. 95$106,2005$.

TENÓRIO, J. A. S; ESPINOSA, D. C. R. Controle Ambiental de Resíduos. In: PHILIPPI JR, A.; ROMÉRO, M. A; BRUNA, G. C. Curso de Gestão Ambiental. Barueri: Manole, 2004.

ZANON, U. Riscos infecciosos imputados ao lixo hospitalar: realidade epidemiológica ou ficção sanitária? Revista Sociedade Brasileira de Medicina Tropical, v. 23, n. 3, p. 163-170, 1990.

\section{Dados dos Autores}

Nome completo: Adeline Chaicouski

Filiação institucional: Universidade Tecnológica Federal do Paraná - Ponta Grossa

Departamento: Coordenação de Alimentos

Função ou cargo ocupado: Graduanda de Tecnologia em Alimentos

Endereço: Rua Adílio Ramos, 198 - Sabará, Ponta Grossa - Paraná, Brasil, 84062-270):

Telefones para contato: (042) 9911-7043 e-mail: chaicouski@ hotmail.com

Nome completo: Joyce Estina da Silva

Filiação institucional: Universidade Tecnológica Federal do Paraná - Ponta Grossa

Departamento: Coordenação de Alimentos

Função ou cargo ocupado: Graduanda de Tecnologia em Alimentos

Endereço: Rua João Cecy Filho, 237, p. 22 - Uvaranas - Ponta Grossa - Paraná, Brasil, 84020-020

Telefones para contato: (042) 9926-0043 e-mail: joyceestina@ hotmail.com

Nome completo: Suélyn Bomfim Nigelski

Filiação institucional: Universidade Tecnológica Federal do Paraná - Ponta Grossa

Departamento: Coordenação de Alimentos

Função ou cargo ocupado: Graduanda de Tecnologia em Alimentos

Endereço: Rua Dr. Julio Prestes de Albuquerque, Ponta Grossa - Paraná, Brasil, 84030-400

Telefones para contato: (042) 9103-3100 e-mail: sunigelski@hotmail.com 
Nome completo: Rosane Natume

Filiação institucional: Universidade Tecnológica Federal do Paraná - Ponta Grossa Departamento: Coordenação de Alimentos

Função ou cargo ocupado: Professora do Curso de Tecnologia em Alimentos

e-mail: rnatume_utfpr@yahoo.com.br

Nome completo: Isonel Sandino Meneguzzo

Filiação institucional: Centro de Ensino Superior dos Campos Gerais - CESCAGE

Departamento: Coordenação de Gestão Ambiental

Função ou cargo ocupado: Graduanda de Tecnologia em Alimentos

Endereço: Rua Adílio Ramos, 198 - Sabará, Ponta Grossa - Paraná, Brasil, 84062-270

Telefones para contato: (042) 9912-1569 e-mail: imeneguzzo@ hotmail.com 